\title{
Efficient Isolation of Cardiac Stem Cells from Brown Adipose
}

\author{
Zhiqiang Liu, Haibin Wang, Ye Zhang, Jin Zhou, Qiuxia Lin, Yanmeng Wang, Cuimi Duan, \\ Kuiwu Wu, and Changyong Wang
}

Department of Tissue Engineering, Institute of Basic Medical Sciences and Tissue Engineering Research Center, Academy of Military Medical Sciences, Beijing 100850, China

Correspondence should be addressed to Kuiwu Wu, ammswu@sina.com and Changyong Wang, wcy2000_te@yahoo.com

Received 30 October 2009; Revised 5 February 2010; Accepted 10 February 2010

Academic Editor: Aikaterini Kontrogianni-Konstantopoulos

Copyright () 2010 Zhiqiang Liu et al. This is an open access article distributed under the Creative Commons Attribution License, which permits unrestricted use, distribution, and reproduction in any medium, provided the original work is properly cited.

Cardiac stem cells represent a logical cell type to exploit in cardiac regeneration. The efficient harvest of cardiac stem cells from a suitable source would turn promising in cardiac stem cell therapy. Brown adipose was recently found to be a new source of cardiac stem cells, instrumental to myocardial regeneration. Unfortunately, an efficient method for the cell isolation is unavailable so far. In our study we have developed a new method for the efficient isolation of cardiac stem cells from brown adipose by combining different enzymes. Results showed that the total cell yield dramatically increased (more than 10 times, $P<.01$ ) compared with that by previous method. The content of CD133-positive cells (reported to differentiate into cardiomyocytes with a high frequency) was much higher than that in the previous report (22.43\% versus 3.5\%). Moreover, the isolated cells could be the efficiently differentiated into functional cardiomyocytes in optimized conditions. Thus, the new method we established would be of great use in further exploring cardiac stem cell therapy.

\section{Introduction}

Despite the notion that adult hearts are terminally differentiated organs without self-renewal potential undermined by the discovery of resident cardiac stem/progenitor cells, the endogenous regenerative mechanisms are too limited to sufficiently compensate for the cardiomyocytes loss occurring in pathological state (e.g., myocardial infarction) [1-3]. This led to numerous investigations to identify a putative source of new cardiomyocytes to ameliorate the injured myocardium and improve the cardiac function. Stem cells, capable of differentiating into other cell types, that is, functional cardiomyocytes, have intrigued intensive studies. So far various stem cell sources have been explored for myocardial regeneration, including embryonic stem, skeletal myoblasts, bone marrow mesenchymal stem cells [4-7]. Data demonstrated functional improvement of the infarcted heart by transplanting these cells. However, the cells also have shortfalls, for example, the potential of tumorigenicity with embryonic stem cells, arrhythmogenicity with skeletal myoblasts, and the controversial transdifferentiation of bone marrow-derived stem cells [8]. These problems underscore the need to search for new sources of adult stem cells to generate cardiomyocytes against the failing myocardium.

The identification and isolation of cardiac stem cells (CSCs) reignited the excitement in this field $[3,9,10]$. Different from other adult stem cells, cardiac stem cells represent a logical source to exploit in myocardial regeneration because of their likelihood to be intrinsically programmed to generate cardiac tissues in vitro and increase its viability in vivo. Therefore, the cardiac stem cell therapy may pioneer an innovative approach to treat heart diseases [11]. However, technical difficulties exist in collecting the cells at present. The number of cells upon harvest is too low $[12,13]$. Therefore, it would be appealing to search for an alternative source of cardiac stem cells.

Adipose tissues are abundant in mammals. Once successfully explored, the tissue source would have far-reaching effects in regenerative medicine $[14,15]$. Therefore, adiposederived cells were also extensively investigated as candidates for the myocardial regeneration by many groups $[16,17]$. Planat-Bénard et al. first reported the spontaneous differentiation of murine adipose-derived cells into cardiomyocytes in 2004 [1]. In spite of its rather low rate $(0.02 \%$ to $0.07 \%)$, 
the cardiomyogenic differentiation suggested that adipose tissue may provide a new source for cardiac progenitor/stem cells. The subsequent studies by Yamada et al. further demonstrated that the cardiac differentiation was far more efficient in brown adipose-derived cells ( $\geqq 20 \%$ ) than that described by Planat-Bénard $[1,12]$. They have proved brown adipose to be an abundant source of cardiac stem cells.

The discovery of the cardiac stem cells in brown adipose opened a new channel to provide cardiomyocytes for myocardial regeneration. However, the previous methods $[1,12]$ for cell isolation are ineffective. In the present study, we developed a new method to isolate brown adiposederived cardiac stem cells with great efficiency by combining collagenase IV and dispase II, with trypsin. The optimized isolation and differentiation of obtained cells were detailed. Moreover, the cardiomyogenic efficiency of isolated cells with this new method was also evaluated.

\section{Materials and Methods}

2.1. Animals and Tissue Samples. All animals were purchased from the Experimental Animal Center, Academy of Military Medical Science (Beijing, China). The Institutional Animal Care and Use Committee (IACUC) of the Chinese Academy of Military Medical Science, Beijing, China, approved all experiments in this study. To obtain tissue samples, animals were killed by intraperitoneally injecting with overdose sodium pentobarbital. The brown adipose tissue was derived from interscapular of neonatal SD rats (Postnatal 7-14 days). Every effort was made to minimize animal suffering and the number of animals used.

2.2. Cell Isolation from Rat Brown Adipose. Isolated brown adipose tissues were washed extensively with sterile phosphate-buffered saline (PBS) to remove contaminating debris and red blood cells. Then it was minced with scissors and digested with $0.1 \%$ collagnase IV (m/v, Sigma), $0.1 \%$ dispase II ( $\mathrm{m} / \mathrm{v}$, Roche Diagnostics, Mannheim, Germany) and $0.05 \%$ trypsin $(\mathrm{m} / \mathrm{v}$, Sigma $)$ in serum-free medium. The digestion was performed at $37^{\circ} \mathrm{C}$ for $30-60$ minutes with gentle agitation. Then, the enzymes were inactivated with an equal volume of $\alpha$-MEM/10\% fetal bovine serum (FBS, Gibco) and the samples were filtered through a $75-\mu \mathrm{m}$ mesh filter to remove debris. The cellular pellets were resuspended in $\alpha$-MEM $/ 10 \%$ fetal bovine serum (FBS); red cell lysis buffer was added. After a 5-minute lysis period, cells were centrifuged and resuspended. Nucleated cells were counted for cell yield. Then cells were plated onto conventional culture plates and cultured under $37^{\circ} \mathrm{C}, 5 \%$ $\mathrm{CO}_{2}$ conditions. For cell viability determination, a portion of cells were stained with propidiumiodide (PI) and analyzed by flow cytometry.

2.3. Flow Cytometry Analysis. For flow cytometry, the newly isolated stromal cells from brown adipose were lyzed by red cell lysis buffer as described above. Then, cells were harvested for flow cytometry analysis as previously described [18]. Briefly, cells were fixed for 30 minutes in ice-cold 2\% paraformaldehyde. The fixed cells were washed in flow cytometry buffer (FBS, Biolegend, 420201) and incubated for 30 minutes in FBS containing the following antibodies: fluorescein isothiocyanate-conjugated antiCD90 (Biolegend, 202503), phycoerythrin-conjugated antiCD29 (Biolegend,102221), FITC-conjugated antiCD45 (Biolegend, 202205), FITC-conjugated CD34 (Santa Cruze, sc-7324), as well as CD133 (Santa Cruze, sc-32596). For unlabelled primary antibodies, FITC-conjugated secondary antibodies were added. All analyses were performed using a BD flow cytometer (BD Bioscience, San Jose, CA).

\subsection{Differentiating CSCs into Cardiomyocytes. CSCs from} brown adipose have the potential to spontaneously differentiate into functional cardiomyocytes as previously reported [12]. To determine the optimal seeding density for cardiomyocytes differentiation, primary stromal cells were seeded onto culture plates at different densities, ranging from $2.5 \times$ $10^{3}$ to $8 \times 10^{4}$ cells $/ \mathrm{cm}^{2}$. The cells were incubated in $37^{\circ} \mathrm{C}$, $5 \% \mathrm{CO}_{2}$ for culture and differentiation. The medium was changed every 2 days. Cells of different seeding densities were observed every day under an inverted phase-contrast microscope (IX70 inverted system microscopy, Olympus Optical, Melville, NY). Number as well as morphology of developing clones was recorded.

2.5. Immunohistochemistry. To investigate the expression of cardiac specific antigens in the CSC-derived cardiomyocytes, immunohistochemical analyses on slides with elongated clusters of cells [19]. Briefly, isolated cells from brown adipose were seeded on slides. The cells were cultured and differentiated for $3-4 \mathrm{w}$ as described above. Then, differentiated cells grown in slides were washed and fixed with $4 \%$ paraformaldehyde in $0.1 \mathrm{M}$ phosphate buffer ( $\mathrm{pH}$ 7.4). After permeabilization with $0.1 \%$ Triton X-100, the cells were incubated with the primary antibody against $\mathrm{cTnT}$ (diluted $1: 200$, Sigma) and $\alpha$-sarcomeric actinin (diluted $1: 200$; Sigma) overnight at $4^{\circ} \mathrm{C}$. FITC-labeled goat antimouse IgG was used as the secondary antibody. The cells were incubated with Hoechst33258 for genomic DNA staining and observed under a fluorescent microscope (Olympus Optical, Melville, $\mathrm{NY})$.

2.6. Pharmacological Studies. Differentiated cells with a regular contractile activity were selected. The basal beating rate was recorded before and after the replacement of culture medium by the fresh $\alpha$ MEM medium (Gibco) containing $10 \%$ of fetal bovine serum. Chronotropic responses were then assessed in $\alpha \mathrm{MEM} / 10 \% \mathrm{FBS}$ by extra-cellular recording of the beating rate in the presence of the appropriate drugs. Dose-response experiments were performed with 0.25 to $5 \times 10^{-6} \mu \mathrm{mol} / \mathrm{L}$ isoproterenol and 5 to $10 \times 10^{-6} \mu \mathrm{mol} / \mathrm{L}$ dilthiazem. Antagonist was added after the maximal dose of agonist. The values were represented as mean \pm SD.

2.7. RNA Extraction and RT-PCR Analysis. For RNA extraction, elongated clusters of cells were dissected under an inverted phase-contrast microscope as previous report [20]. 
TABle 1: Correlation between digest time and cell yield when different enzymes were used for brown adipose digest.

\begin{tabular}{|c|c|c|c|c|}
\hline \multicolumn{5}{|c|}{ Digest time-Cell yield } \\
\hline Time & Enzyme* & Cell yield $\$$ & Cell viability & Viable cell yield ${ }^{\$}$ \\
\hline \multirow{5}{*}{30 minutes } & $\mathrm{D} / \mathrm{IV} / \mathrm{T}$ & $8.49 \pm 0.57$ & $93.47 \pm 1.18 \%$ & $7.94 \pm 0.58$ \\
\hline & $\mathrm{IV} / \mathrm{T}$ & $2.74 \pm 0.39$ & $93.79 \pm 1.22 \%$ & $2.57 \pm 0.39$ \\
\hline & IV & $1.47 \pm 0.19$ & $94.66 \pm 0.99 \%$ & $1.39 \pm 0.18$ \\
\hline & I & $0.18 \pm 0.02$ & $93.95 \pm 1.05 \%$ & $0.17 \pm 0.02$ \\
\hline & $\mathrm{D}$ & $0.78 \pm 0.07$ & $94.96 \pm 0.88 \%$ & $0.74 \pm 0.07$ \\
\hline \multirow{5}{*}{45 minutes } & $\mathrm{D} / \mathrm{IV} / \mathrm{T}$ & $11.65 \pm 1.07$ & $90.72 \pm 1.38 \%$ & $10.56 \pm 0.98^{\#}$ \\
\hline & $\mathrm{IV} / \mathrm{T}$ & $4.26 \pm 0.56$ & $91.87 \pm 1.34 \%$ & $3.92 \pm 0.54$ \\
\hline & IV & $1.82 \pm 0.20$ & $92.87 \pm 1.69 \%$ & $1.69 \pm 0.20$ \\
\hline & I & $0.23 \pm 0.04$ & $91.96 \pm 0.77 \%$ & $0.22 \pm 0.04$ \\
\hline & $\mathrm{D}$ & $0.87 \pm 0.06$ & $93.18 \pm 1.36 \%$ & $0.81 \pm 0.07$ \\
\hline \multirow{5}{*}{60 minutes } & $\mathrm{D} / \mathrm{IV} / \mathrm{T}$ & $11.72 \pm 0.86$ & $86.88 \pm 1.50 \%$ & $10.19 \pm 0.91$ \\
\hline & $\mathrm{IV} / \mathrm{T}$ & $4.66 \pm 0.33$ & $90.86 \pm 1.45 \%$ & $4.23 \pm 0.31^{\#}$ \\
\hline & IV & $1.96 \pm 0.15$ & $91.17 \pm 1.55 \%$ & $1.79 \pm 0.14^{\#}$ \\
\hline & I & $0.28 \pm 0.04$ & $90.10 \pm 1.14 \%$ & $0.26 \pm 0.04^{\#}$ \\
\hline & $\mathrm{D}$ & $1.02 \pm 0.13$ & $91.14 \pm 1.57 \%$ & $0.93 \pm 0.12^{\#}$ \\
\hline
\end{tabular}

Nucleated cell yields of cell viability were compared among different enzymes and different digest time. Data are presented as mean \pm standard deviation of $n=6$ for each group.

*D Dispase II; IV: collagenase IV; T: trypsin; I: collagenase I;

\# Optimal cell yield for the enzyme.

$\$ 10^{6}$ cells/g tissue.

Total RNA was extracted with RNAprep pure Cell/Bacteria Kit (TIANGEN) according to manufacturer's instruction. Reverse transcription were performed using standard procedures to synthesize first-strand cDNA. The gene-specific primers for cTnI (5' -CTCGGAGTATCAGGAAGAGCACA; 3'-TAAACTTGCCACGCAGGTCATAG, 216bp), GATA4 (5' -CTGTCATCTCACTATGGGCA;3'-CCAAGTCCGAGCAGGAATT, 257 bp), Nkx2.5 (5'-CAGTGGAGCTGGACAAAGCC; 3'-TAGCGACGGTTCTGGAACCA, 216 bp), MEF2C ( $5^{\prime}$-AGCAAGAATACGATGCCATC; $3^{\prime}$-GAAGGGGTGGTGGTACGGTC, $347 \mathrm{bp}), \beta$-Actin ( $5^{\prime}$-AGAGGGAAATCGTGCGTGAC; $3^{\prime}$-AGGAGCCAGGCAGTAATC, $353 \mathrm{bp}$ ) were used in the following PCR amplification. Each cycle consisted of denaturation at $94^{\circ} \mathrm{C}$ for 30 secondes, and annealing/extension at $72^{\circ} \mathrm{C}$ for 45 secondes.

2.8. Statistical Analysis. The data are reported as means \pm SD. Multiple related samples were compared using the Friedman test, results of which were complemented with posthoc pairwise analysis by Wilcoxon signed-rank test. Statistical analyses was performed with SPSS 11.0 (SPSS, Inc., Chicago, IL). Significance was accepted at $P<.05$.

\section{Results}

3.1. Optimized Isolation and High Cell Yield. To optimize isolating conditions for cell yield as high as possible, tissue digest was performed in $37^{\circ} \mathrm{C}, 5 \% \mathrm{CO}_{2}$ incubator. The correlation between digesting time and cell yield was investigated, so did cell viability. Comparisons have been made
TABLe 2: Phenotypic characterization of rat brown adipose-derived cells $(n=6)$.

\begin{tabular}{lc}
\hline Surface marker & Positive cells (\%) \\
\hline CD90 & $54.82 \pm 3.50$ \\
CD133 & $22.43 \pm 2.13$ \\
CD29 & $18.23 \pm 1.97$ \\
CD29/CD133 & $8.22 \pm 1.11$ \\
CD45 & $2.09 \pm 0.72$ \\
CD34 & $0.12 \pm 0.05$ \\
\hline
\end{tabular}

against the established method to isolate brown adiposederived cardiac progenitor/stem cells with Dispase II and the classic one for stromal cell isolation from adipose tissue with collagenase I. In addition, other collagenases beside collagenase I were also tried. Collagenase IV was found more efficient than collagenase I for rat adipose digest (Table 1, Figure 1(a)). Thus, collagenase IV was selected for the enzyme combination in the experiment ensued. Data show that cell yield was up to7.94 $\pm 0.58 \times 10^{6} / \mathrm{g}$ tissue when digesting for 30 minutes using the combined enzymes consisting of $0.1 \%$ collagenase IV, $0.1 \%$ dispase II and $0.05 \%$ trypsin. With the prolonged digest, both cell yield and cell death rate were increased (Table 1). When digesting time was prolonged to 60 minutes, cell yield did not significantly increase compared with that of 45 minutes, but cell death rate was significantly increased (up to $13.12 \%$, Figure 1(b)). The highest viable cell yield was obtained at the digesting time of 45 minutes (more than 107 cells/g tissue), while cell viability was maintained up to $\sim 91 \%$. The data show that cell yield was dramatically enhanced compared with previously described method (Figure $1(\mathrm{a}), P<.01$ ) by combining collagenase IV, dispase II and trypsin for brown adipose tissue digest.

3.2. High Cardiomyogenic Potential of Isolated Cells and Cardiac Differentiation. To understand the characteristics of stromal cells isolated from rat brown adipose, we analyzed several surface markers with fluorescence-activated cell sorting (FACS) analyses (Figure 2, Table 2). The expression of CD29, CD90 (suggested to be one of MSC markers), is $18.23 \pm 1.97 \%$ and $54.82 \pm 3.50 \%$, respectively [21]. Most of the cells were negative for CD45, a hematopoietic and leukocyte marker. They also expressed negative for CD34, a myeloid progenitor cell antigen present in endothelial cells and some fibroblasts too [22]. In addition, the percentage of CD133-positive cells was up to $\sim 22.43 \%$. Such cells were confirmed by the previous report [23] to differentiate into cardiomyocytes with a high incidence. The percentage is significantly higher than the previous report $[12,23]$ (which only obtained 3.5\% CD133-positive cells in total brown adipose-derived cells). Among the CD133 positive cells, about $8.22 \pm 1.11 \%$ cells simultaneously express CD29.

After seeding on culture plates at different densities, cells adhered and proliferated, while cardiac stem cells began to differentiate with prolonged culture. As shown in Figure 3, around 6 days of culture, emergence of various 


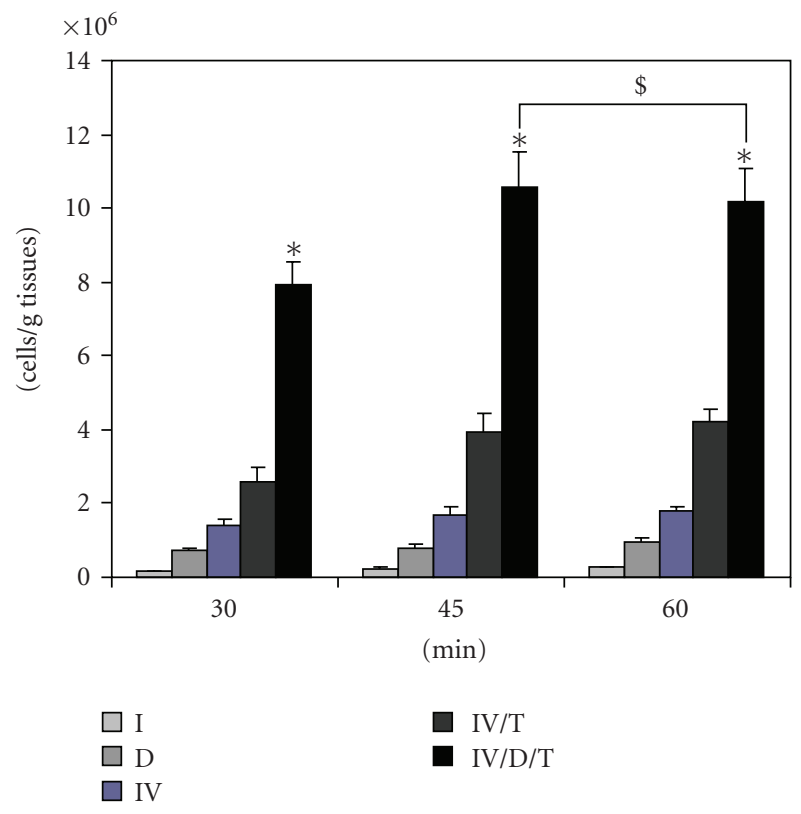

(a)
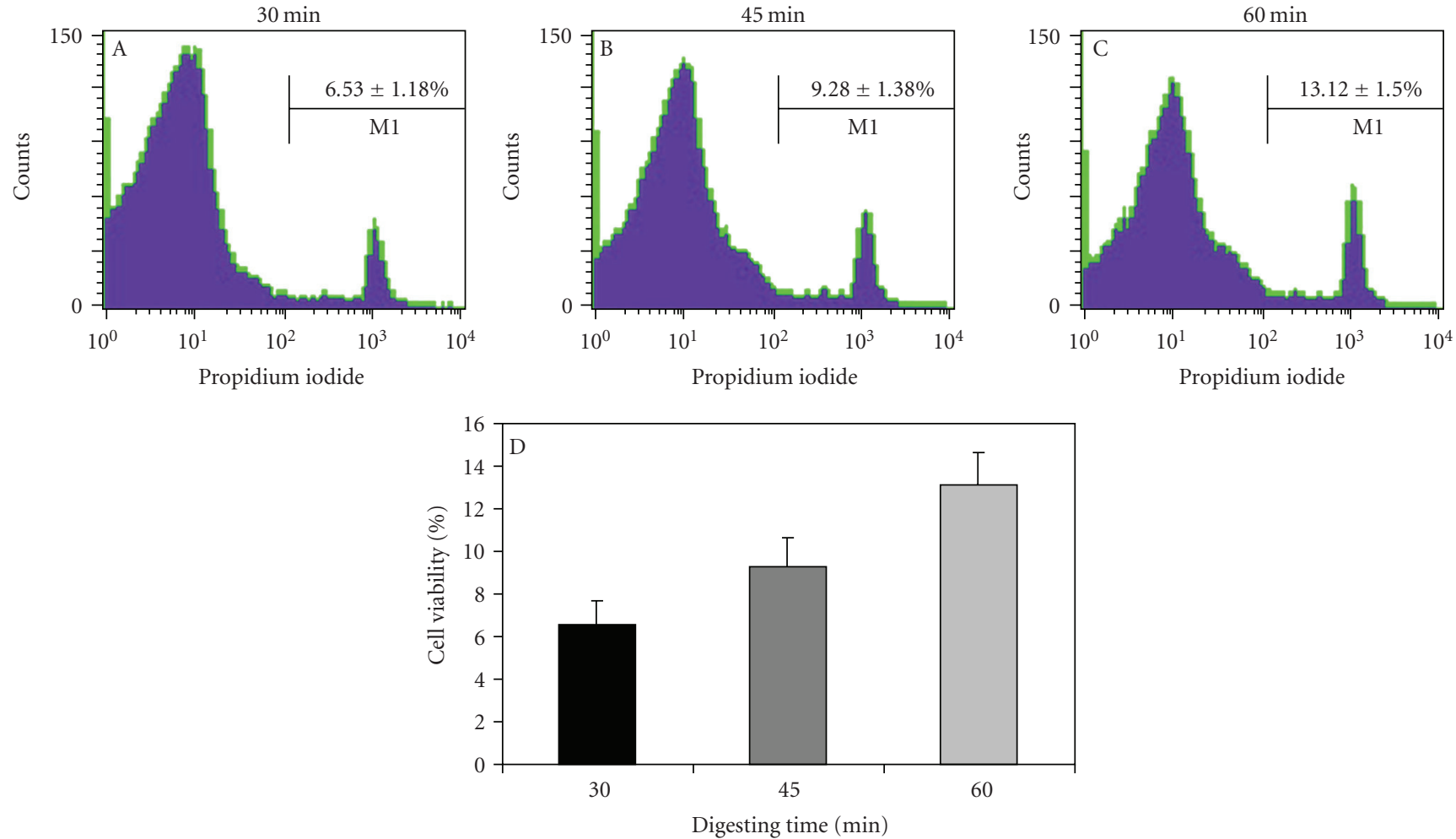

(b)

FIGURE 1: (a) Comparisons between different methods in nucleated cell yield within one hour's digest ( $n=6$ for each group). \$: no significant difference, $P>.05 ;^{*}: P<.01$ compared with all other groups in the same digest time. (b) Representative images of cell viability analysis. Newly isolated cells from rat brown adipose were stained with Propidium iodide and then analyzed using flow cytometry. A: Cell viability after 30 minutes digestion with collagenase IV, dispase II, and trypsin; B: cell viability after 45 minutes digestion with collagenase IV, dispase II, and trypsin; C: cell viability after 60 minutes digestion with collagenase IV, dispase II, and trypsin; D: Comparison of cell viabilities after digesting for different time with collagenase IV, dispase II, and trypsin, ( $n=6$ for each group). 


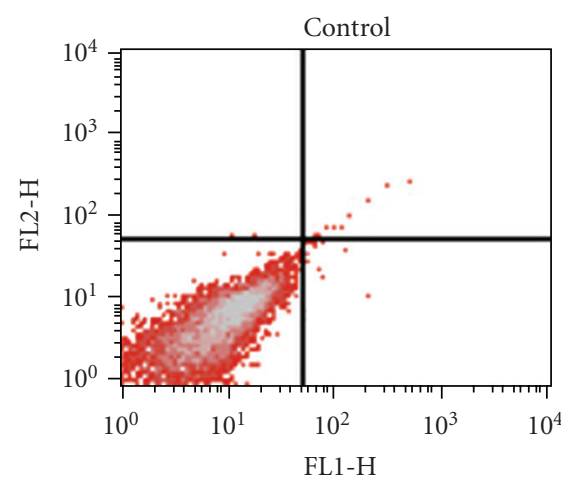

(a)

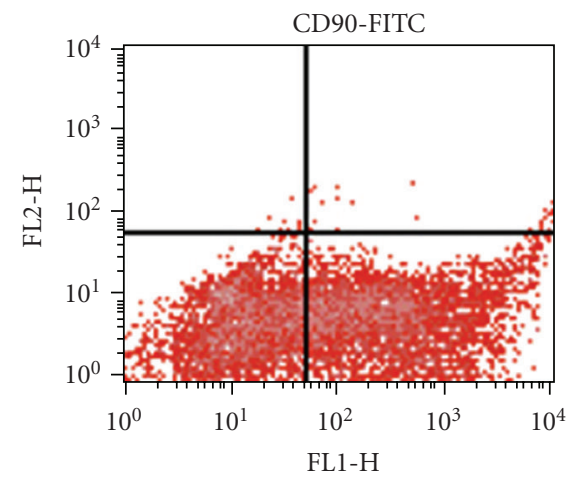

(b)

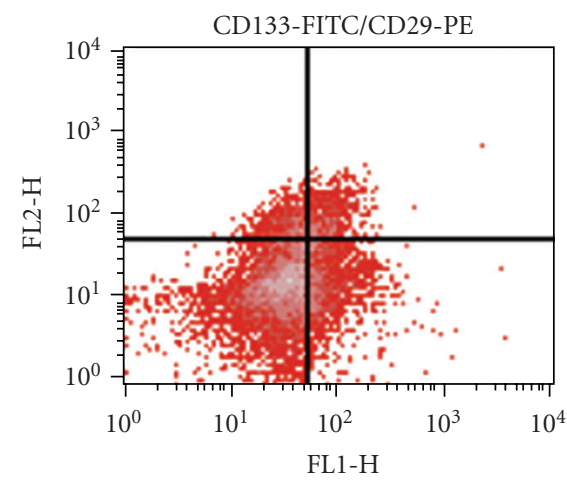

(c)

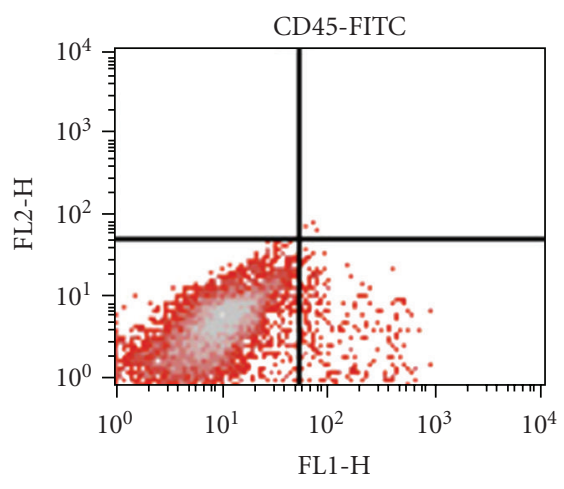

(d)

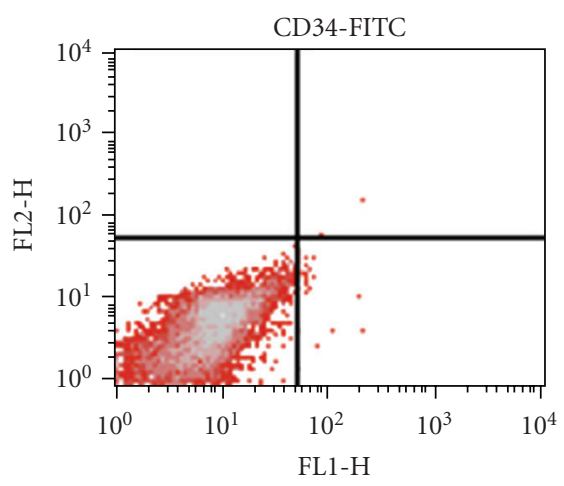

(e)

FIGURE 2: Representative images of flow cytometry analyses for rat brown adipose-derived cells. Brown adipose-derived cells are partly positive for CD-29, CD90, and CD133, while negative for CD45 and CD34. (a) Control; (b) Flow cytometry analysis for surface marker CD90; (c) Flow cytometry analysis for surface marker CD29 and CD133; (d) Flow cytometry analysis for surface marker CD45; (e) Flow cytometry analysis for surface marker CD34.

cell morphologies were identified, that is, fibroblast-like appearance, clusters of preadipocytes/adipocytes, and clones of rounded cells together with small tube cells. At 1 week, elongated cells appeared and started a contractile activity independently. Following this, increasingly more elongated morphology and contractile cells appeared with the time (Figure 3). In 3 to 4 weeks, groups of contractile cells could be seen in nearly any field under an inverted phase-contrast microscope. In the whole process, differentiation proceeded spontaneously, using no inductor. However, the percentage of beating cells counted at 3-4 weeks varied among the seeding densities of isolated cells (details below).

\subsection{CSC-Derived Cardiomyocytes Demonstrate Characteristic Similar to Functional Cardiomyocytes}

3.3.1. Expression of Cardiac Markers. In order to define the phenotype of differentiated cells, immunofluorescent staining of cardiac-specific antigens was performed on the slides with elongated clusters of cells. In addition, the expression of cardiac genes was analyzed by RT-PCR. As expected, a specific positive staining was obtained with antibodies against the sarcomeric $\alpha$-actinin (Figure 4(a) A-C) and cardiac troponin $\mathrm{T}$ (Figure 4(a) E-G); even the transverse striation can be clearly observed (Figure 4(a) D, H). Some elongated cells that do not contract under conditions could also be stained by $\mathrm{cTnT} / \alpha$-actinin. In contrast, antibodies against the skeletal muscle protein MyoD (data not show) did not stain the elongated cells. The expressions of cTnI, GATA4, Nkx2.5, MEF2C were also verified (Figure 4(b)). These data strongly argued for the cardiomyocyte nature of the contracting cells.

3.3.2. Reaction with Pharmacologic Reagents. To test the functionality of CSC-derived cardiomyocytes, pharmacological studies were performed at 28 days after differentiation of brown adipose-derived CSCs. Several pharmacologic agents known to influence the heart rate were added and the chronotropic responses of contracting cells to these agents were examined. As expected for differentiated cardiomyocytes, the $\beta$-agonist isoproterenol induced a dose-dependent increase of the spontaneous contraction rate measured as beatings per minute (Figure 5). Diltiazem, a nonselective $\beta$-adrenergic antagonist, reversed the isoproterenol-induced acceleration (Figure 5). These chronotropic responses definitively demonstrated that these cells responded like functional cardiomyocytes. Note that there was a great heterogeneity in the sensitivity to these heart pharmacologic agents among different cardiomyocytes. For instance, some cardiomyocytes that did not contract 


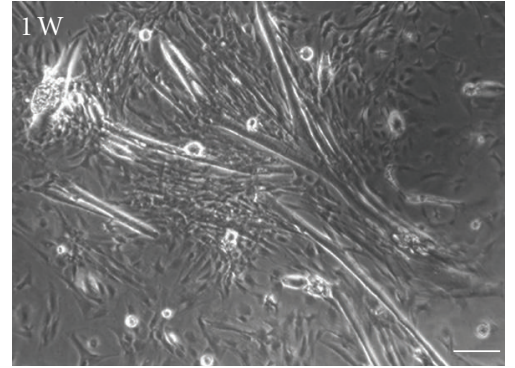

(a)

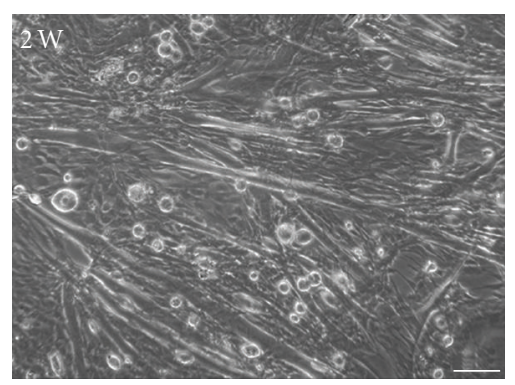

(d)

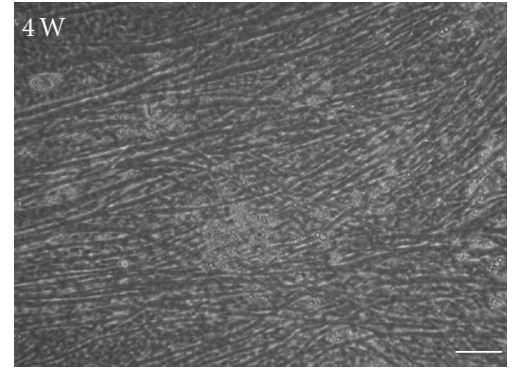

(g)

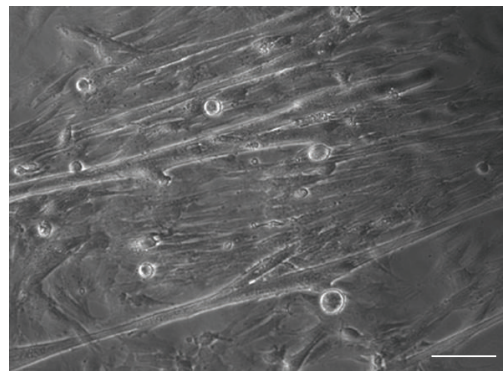

(b)

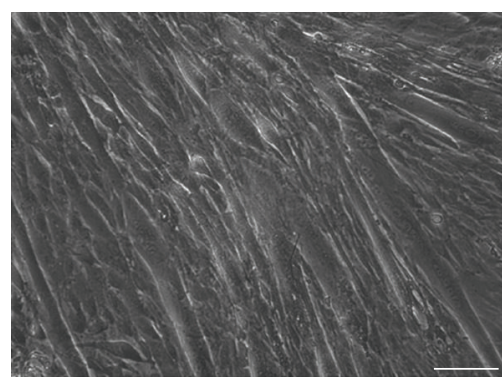

(e)

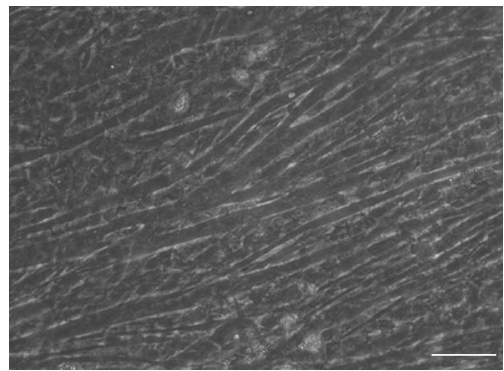

(h)

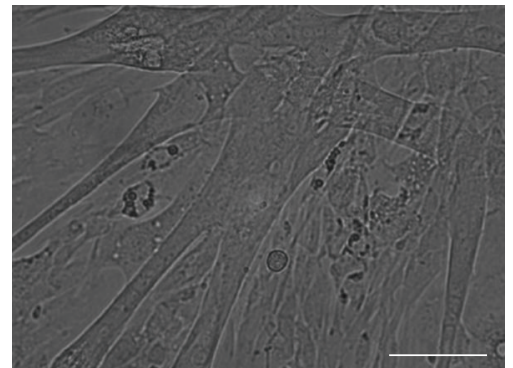

(c)

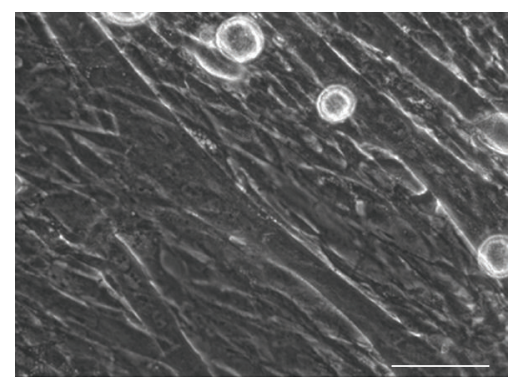

(f)

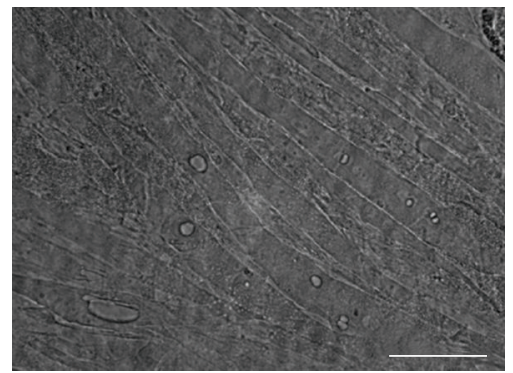

(i)

FIGURE 3: Culture and differentiation of brown adipose-derived cardiac stem cells. Phase-contrast microscopy of differentiated cardiomyocyteslike cells at days 7, 14, and 28. Isolated primary cells were plated into culture dished $\left(2.5 \times 10^{4} \mathrm{cells} / \mathrm{cm}^{2}\right)$ and observed every day under an inverted phase-contrast microscope at (a)-(c) 1 weeks after spontaneous differentiation, (d)-(f) 2 weeks after spontaneous differentiation, (g)-(i) 4 weeks after spontaneous differentiation. Differentiated cells gradually increased in size with time due to cell proliferation. Early, differentiated cells were rare in plates (day 7), then elongated cells appeared (day 14 and 28) more and more, and they could spontaneously beat like functional cardiomyocytes (bar $=100 \mu \mathrm{m})$.

TABLE 3: Comparison of differentiated cTnT+ cardiomyocytes (\%) from brown adipose-derived cells at different seeding densities.

\begin{tabular}{lcccccc}
\hline Cell density & $5 \times 10^{4} / \mathrm{cm}^{2}$ & $2.5 \times 10^{4} / \mathrm{cm}^{2}$ & $1 \times 10^{4} / \mathrm{cm}^{2}$ & $5 \times 10^{3} / \mathrm{cm}^{2}$ & $2.5 \times 10^{3} / \mathrm{cm}^{2}$ & $1 \times 10^{3} / \mathrm{cm}^{2}$ \\
\hline Combination & $24.78 \pm 2.4$ & $29.93 \pm 3.2^{\#}$ & $25.41 \pm 2.23$ & $21.43 \pm 1.62$ & $16.7 \pm 1.41$ & $5.3 \pm 0.6$ \\
Dispase II* & $11.64 \pm 1.98$ & $15.43 \pm 2.76$ & $12.35 \pm 2.21$ & $9.64 \pm 2.55$ & $5.7 \pm 1.24$ & $2.41 \pm 0.91$ \\
\hline
\end{tabular}

* Combination: cells isolated by combination of collgenase IV, dispase II, and trypsin; Dispase II: cells isolated by dispase II alone.

\#The optimal seeding density for brown adipose-derived cardiac stem cells differentiation into cardiomyocytes $(P<.01, n=6)$.

under control conditions started to beat under $\beta$-adrenergic stimulation (data not shown). However, when cells were responsive, similar pattern toward the drugs was also observed.

\subsection{Optimal Seeding Density for Cardiac Stem Cell Differ-} entiation. The primary seeding density of brown adiposederived cells influences the differentiation efficiency of cardiac stem cells into cardiomyocytes, as was demonstrated in the previous report [12]. So correlation between seeding density of primary cells and percentage of differentiated cardiomyocytes was investigated to achieve the optimal cardiac differentiation. As shown in Table 3, the appearance of cTnT $^{+}$CM cells varies from $\sim 5.3 \pm 0.6 \%$ to $\sim 29.93 \pm 3.2 \%$ with the primary seeding densities increasing from $1 \times$ $10^{3} / \mathrm{cm}^{2}-5 \times 10^{4} / \mathrm{cm}^{2}$. These data showed that cells we isolated from brown adipose by using this new method have even higher cardiomyogenic potential than that previously 

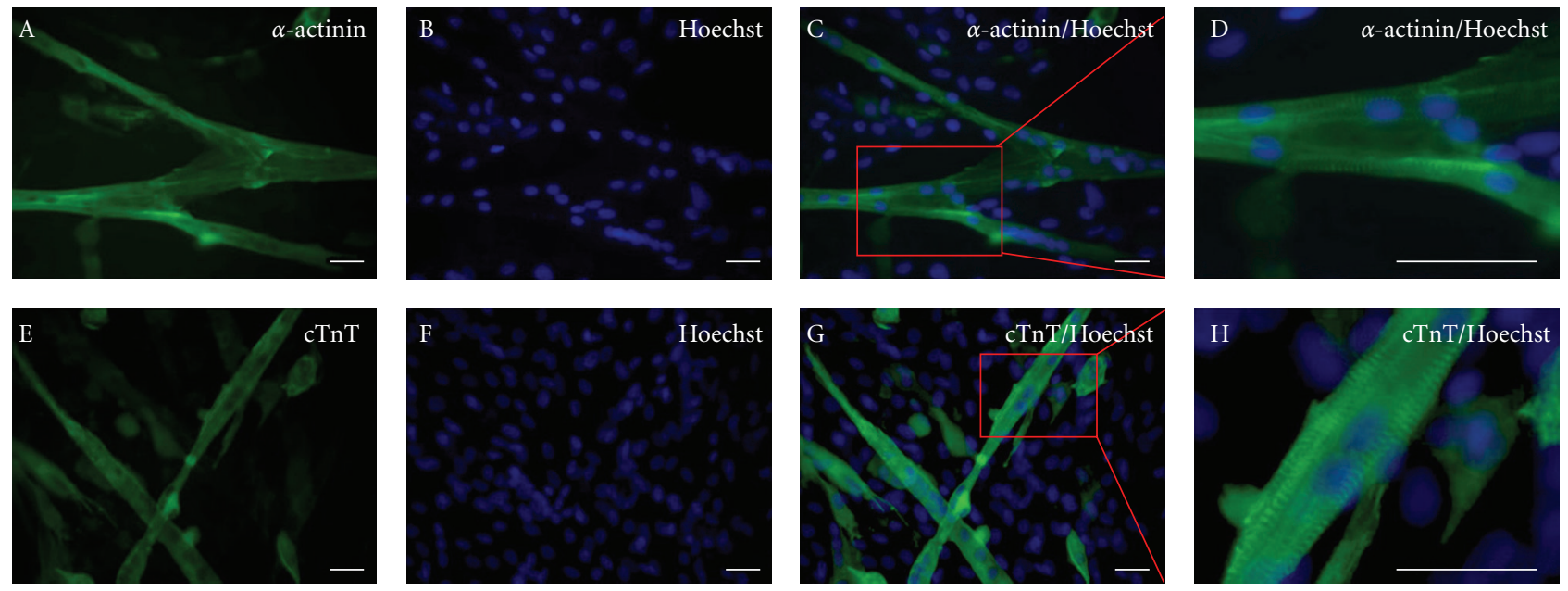

(a)

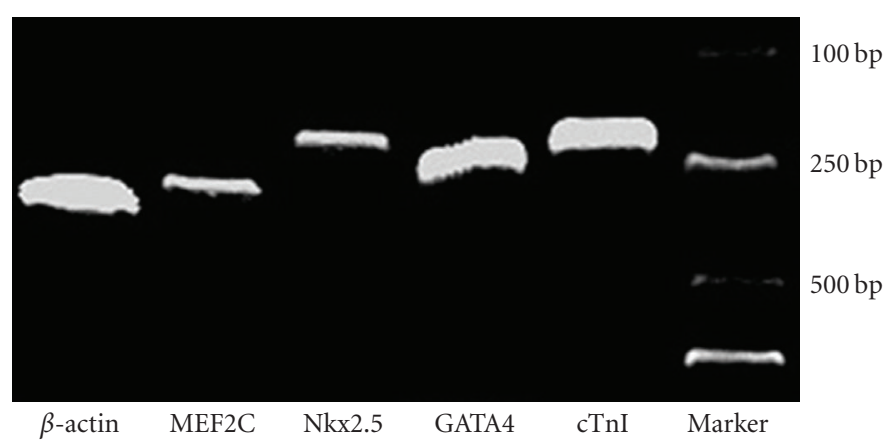

(b)

FIgURE 4: (a) Immunostaining of differentiated cells for cardiac-specific antibodies. Differentiated cardiomyocytes-like cells were specifically stained with anti $\alpha$-sarcomeric actinin A-D, and anticardiac troponin A-I antibodies; the transverse striation can be clearly observed under high magnification microcopy (D and I, 4 magnifications of red frame in C and G, resp.). Furthermore, no specific staining was obtained with the antiMyoD (data not shown) $(\mathrm{Bar}=50 \mu \mathrm{m})$. (b) Expression of cardiac-specific genes in the differented cells analyzed by RT-PCR.

reported [12]. Note that no sorting procedure was performed in our study (also different from the previous report) [12].

\section{Discussion}

Heart disease is the leading cause of death in the industrial world. The stem cell therapy seems to be a promising treatment for injured myocardium. To reach this goal, it is desirable to find a good source of stem cells that can be used to obtain new myocardium on time [21]. Several cell types, for example, ESC, BMMSC, skeletal myoblasts, have been considered candidates for myocardial regeneration. However, each of them has shortfalls in clinical applications [4-8]. The best candidates seem to be cardiac "progenitor" and/or "stem" cells [21]. Currently the cardiac stem cell transplantation for regenerating infarcted myocardium has invited a great deal of interest. Many laboratories have attempted to isolate potential cardiac stem cells from several tissues [22]. In this study, we firstly combined collagenase and dispase II with trypsin for brown adipose digestion to isolate cardiac stem cells. Our main findings are the following: (1) We established a new method to efficiently isolate cells from rat brown adipose with a high content of CSCs, and total cell yield increased up to more than $10^{7}$ /gram tissue. (2) We corroborated brown adipose to be an abundant source of cardiac stem cells, useful for in vitro studies of cardiomyocytes differentiation/(patho) physiology and in vivo studies of cardiac stem cell therapy.

We demonstrated that a single collagenase or dispase II was insufficient for brown adipose digestion, leading to relative lower cell yield (Figure $1(\mathrm{a})$ ). However, when collagenase was combined with dispase II supplemented by trypsin, cell yield dramatically increased by manifolds (Figure 1(a)). Efficient tissue digestion was also observed. I our experiment, tissue blocks also left undigested when collagenase or dispase II was applied alone, albeit digesting time extended to more than 2 hours (data not shown). In contrast, tissue blocks could be hardly found in 30 minutes digestion by combining three enzymes, indicating optimally sufficient tissue digestion.

Several years ago, a single protease was demonstrated to have limitations in digesting capacity when applied to digest adipose tissues. Different protease activity was necessary for optimal tissue digest efficacy and cell yield [19, 24-26]. 


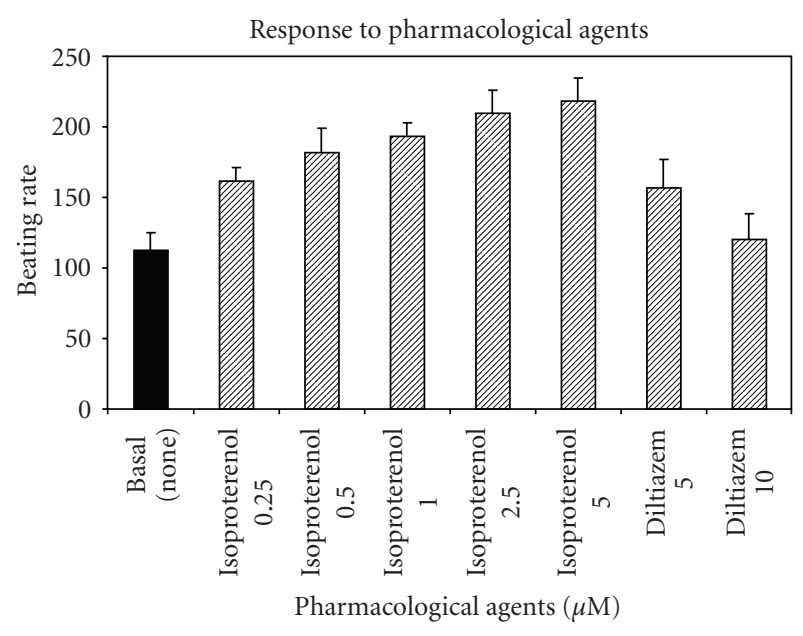

FIGURE 5: Chronotropic response of contracting cells to adrenergic and dilthiazem stimulation. Isolated cells were plated into 6-well plate for 28 days. Basal beating rate was recorded. Contracting rate was measured by the treatment with the $\beta$-adrenergic agonist isoproterenol and antagonist dilthiazem. Results represent the mean of 6 to 10 separate experiments.

However, the challenge is to balance the combination of enzymes and digesting time for the desired cell yield and function [27]. Additionally, it has been shown that parameters (e.g., digest time and enzyme activity) applied in the isolating procedure of primary cells have a significant effect on cell yield, viability, and phenotype. Therefore, isolating conditions were optimized. Isolated cells were also evaluated for cardiomyogenic potential in our study. The tissue was digested in $37^{\circ} \mathrm{C}$, pH7.2-7.4, favorable for enzyme activities. A magnetic stirrer was used to facilitate tissue digestion. Under these conditions, optimal digesting time was determined to be 45 minutes (to balance the total cell yield and viability). When differentiating obtained cells into cardiomyocytes, the isolated cells by this new method were of high cardiomyogenic potential compared with what was previously described [12]. Thus, this new method is proved efficient in total cell yield from brown adipose with high cardiomyogenic potential. It is noteworthy that the method is also suitable for multipotent stem cell isolation from rat white adipose tissues (data not shown).

In consistency with the previous reports $[12,23]$, we failed to get purified cardiac stem cells from brown adiposederived cell population. Thus a valid procedure for their expansion in undifferentiated states was not established. To further their applications in cell therapies, a technique for rapid purification as well as a method of clinical grade expansion for the cardiac stem cells is needed.

\section{Conclusion}

In this study, we have found an optimal combination of digest enzymes consisting of $0.1 \%$ collagenase IV, $0.1 \%$ dispase II, and $0.05 \%$ trypsin for efficient isolation of cardiac stem cells from brown adipose tissues. The procedure was optimized for maximum viable cell yield (up to $10^{7} /$ cells from 1 gram tissue sample). Furthermore, the obtained cells could be effectively differentiated into functional cardiomyocytes. Therefore, we established a novel and efficient method for cardiac stem cell isolation from brown adipose. It will pave the way for cardiac stem cell therapy as well as in basic research.

\section{Acknowledgment}

This work was supported by the National High Technology Research and Development Program of China (no. 2006AA02A105), the Nature Science Foundation of China (no. 30530220), Beijing Nature science Foundation of China (no. 7062053). The authors thank C. Chen for technical assistance in histological preparation and staining Z. liu, $\mathrm{H}$. Wang, Y. Zhang, and J. Zhou contributed equally to this work.

\section{References}

[1] V. Planat-Bénard, C. Menard, M. André, et al., "Spontaneous cardiomyocyte differentiation from adipose tissue stroma cells," Circulation Research, vol. 94, no. 2, pp. 223-229, 2004.

[2] W. H. Zimmermann, I. Melnychenko, G. Wasmeier, et al., "Engineered heart tissue grafts improve systolic and diastolic function in infarcted rat hearts," Nature Medicine, vol. 12, no. 4, pp. 452-458, 2006.

[3] A. P. Beltrami, L. Barlucchi, D. Torella, et al., "Adult cardiac stem cells are multipotent and support myocardial regeneration," Cell, vol. 114, no. 6, pp. 763-776, 2003.

[4] T. Kofidis, D. R. Lebl, E. C. Martinez, G. Hoyt, M. Tanaka, and R. C. Robbins, "Novel injectable bioartificial tissue facilitates targeted, less invasive, large-scale tissue restoration on the beating heart after myocardial injury," Circulation, vol. 112, no. 9, supplement, pp. I.173-I.177, 2005.

[5] K. L. Christman, A. J. Vardanian, Q. Fang, R. E. Sievers, H. H. Fok, and R. J. Lee, "Injectable fibrin scaffold improves cell transplant survival, reduces infarct expansion, and induces neovasculature formation in ischemic myocardium," Journal of the American College of Cardiology, vol. 44, no. 3, pp. 654660, 2004

[6] A. A. Mangi, N. Noiseux, D. Kong, et al., "Mesenchymal stem cells modified with Akt prevent remodeling and restore performance of infarcted hearts," Nature Medicine, vol. 9, no. 9, pp. 1195-1201, 2003.

[7] R. Passier, L. W. van Laake, and C. L. Mummery, "Stem-cellbased therapy and lessons from the heart," Nature, vol. 453, no. 7193, pp. 322-329, 2008.

[8] Z. Li, A. Lee, M. Huang, et al., "Imaging survival and function of transplanted cardiac resident stem cells," Journal of the American College of Cardiology, vol. 53, no. 14, pp. 1229-1240, 2009.

[9] A. G. Latronico, M. Coletta, E. Vivarelli, et al., "Isolation and expansion of adult cardiac stem cells from human and murine heart," Circulation Research, vol. 95, no. 9, pp. 911-921, 2004.

[10] A. Linke, P. Müller, D. Nurzynska, et al., "Stem cells in the dog heart are self-renewing, clonogenic, and multipotent and regenerate infarcted myocardium, improving cardiac function," Proceedings of the National Academy of Sciences of the United States of America, vol. 102, no. 25, pp. 8966-8971, 2005. 
[11] L. Barile, I. Chimenti, R. Gaetani, et al., "Cardiac stem cells: isolation, expansion and experimental use for myocardial regeneration," Nature Clinical Practice Cardiovascular Medicine, vol. 4, no. 1, pp. S9-S14, 2007.

[12] Y. Yamada, X.-D. Wang, S.-I. Yokoyama, N. Fukuda, and N. Takakura, "Cardiac progenitor cells in brown adipose tissue repaired damaged myocardium," Biochemical and Biophysical Research Communications, vol. 342, no. 2, pp. 662-670, 2006.

[13] A. M. Smits, P. van Vliet, C. H. Metz, et al., "Human cardiomyocyte progenitor cells differentiate into functional mature cardiomyocytes: an in vitro model for studying human cardiac physiology and pathophysiology," Nature Protocols, vol. 4, no. 2, pp. 232-243, 2009.

[14] G. Loffler and H. Hauner, "Adipose tissue development: the role of precursor cells and adipogenic factors. Part II: the regulation of the adipogenic conversion by hormones and serum factors," Klinische Wochenschrift, vol. 65, no. 17, pp. 812-817, 1987.

[15] J. M. Gimble, A. J. Katz, and B. A. Bunnell, "Adipose-derived stem cells for regenerative medicine," Circulation Research, vol. 100, no. 9, pp. 1249-1260, 2007.

[16] Y. Miyahara, N. Nagaya, K. Masaharu, et al., "Monolayered mesenchymal stem cells repair scarred myocardium after myocardial infarction," Nature Medicine, vol. 12, no. 4, pp. 459-465, 2006.

[17] M. Mazo, V. Planat-Bénard, G. Abizanda, et al., “Transplantation of adipose derived stromal cells is associated with functional improvement in a rat model of chronic myocardial infarction," European Journal of Heart Failure, vol. 10, no. 5, pp. 454-462, 2008.

[18] P. A. Zuk, M. Zhu, P. Ashjian, et al., "Human adipose tissue is a source of multipotent stem cells," Molecular Biology of the Cell, vol. 13, no. 12, pp. 4279-4295, 2002.

[19] S. Lü, S. Liu, W. He, et al., "Bioreactor cultivation enhances NTEB formation and differentiation of NTES cells into cardiomyocytes," Cloning and Stem Cells, vol. 10, no. 3, pp. 363-370, 2008.

[20] B. Léobon, J. Roncalli, C. Joffre, et al., "Adipose-derived cardiomyogenic cells: in vitro expansion and functional improvement in a mouse model of myocardial infarction," Cardiovascular Research, vol. 83, no. 4, pp. 757-767, 2009.

[21] V. Di Felice, A. De Luca, M. L. Colorito, et al., "Cardiac stem cell research: an elephant in the room?" Anatomical Record, vol. 292, no. 3, pp. 449-454, 2009.

[22] J. Pouly, P. Bruneval, C. Mandet, et al., "Cardiac stem cells in the real world," Journal of Thoracic and Cardiovascular Surgery, vol. 135, no. 3, pp. 673-678, 2008.

[23] Y. Yamada, S.-I. Yokoyama, X.-D. Wang, N. Fukuda, and N. Takakura, "Cardiac stem cells in brown adipose tissue express CD133 and induce bone marrow nonhematopoietic cells to differentiate into cardiomyocytes," Stem Cells, vol. 25, no. 5, pp. 1326-1333, 2007.

[24] P. Georges, R. P. Muirhead, L. Williams, et al., "Comparison of size, viability, and function of fetal pig islet-like cell clusters after digestion using collagenase or liberase," Cell Transplantation, vol. 11, no. 6, pp. 539-545, 2002.

[25] H. Wang, C. A. Van Blitterswijk, M. Bertrand-De Haas, A. H. Schuurman, and E. N. Lamme, "Improved enzymatic isolation of fibroblasts for the creation of autologous skin substitutes," In Vitro Cellular and Developmental Biology-Animal, vol. 40, no. 8-9, pp. 268-277, 2004.

[26] D. M. Hayman, T. J. Blumberg, C. C. Scott, and K. A. Athanasiou, "The effects of isolation on chondrocyte gene expression," Tissue Engineering, vol. 12, no. 9, pp. 2573-2581, 2006.

[27] L. Pilgaard, P. Lund, J. G. Rasmussen, T. Fink, and V. Zachar, "Comparative analysis of highly defined proteases for the isolation of adipose tissue-derived stem cells," Regenerative Medicine, vol. 3, no. 5, pp. 705-715, 2008. 

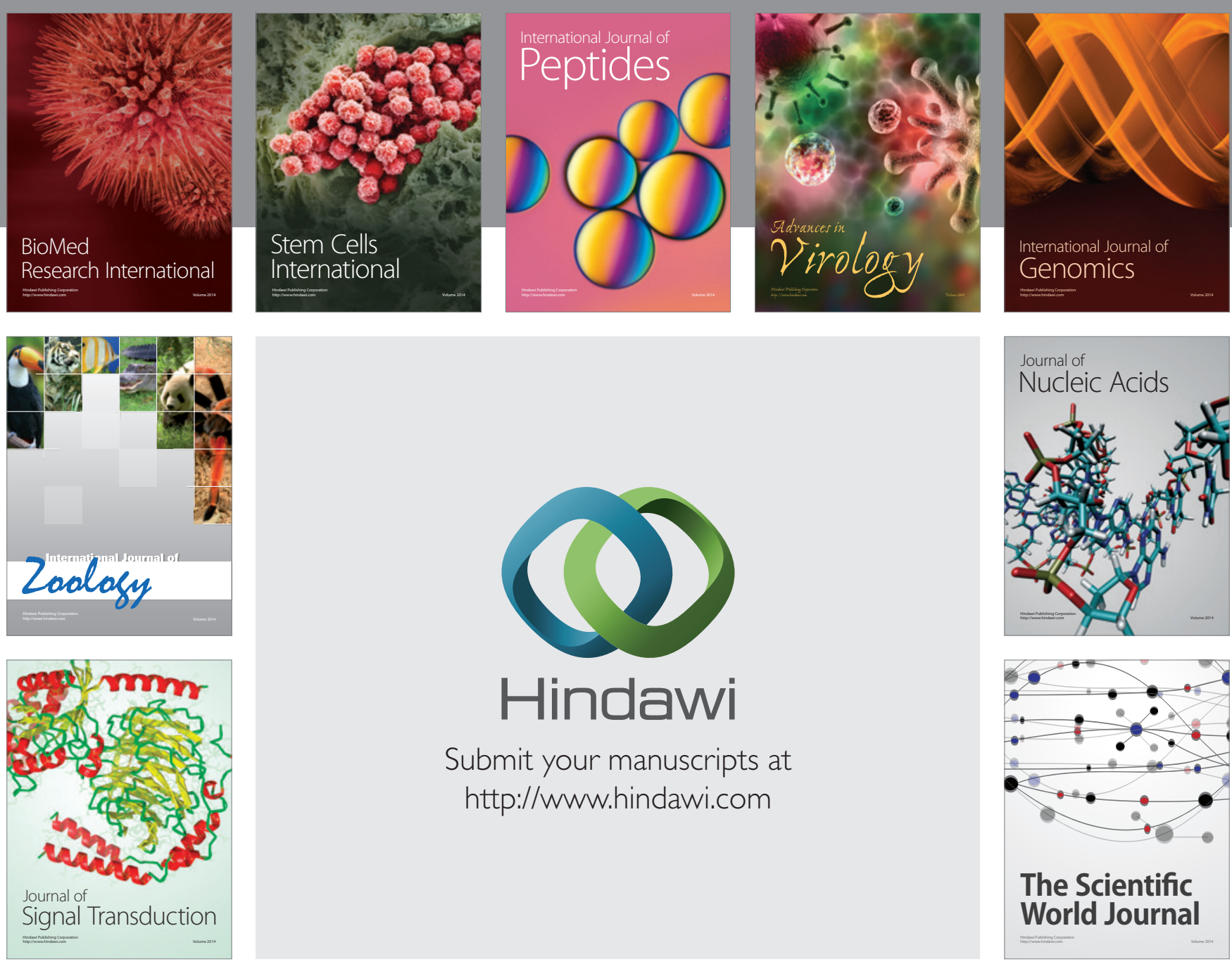

Submit your manuscripts at

http://www.hindawi.com
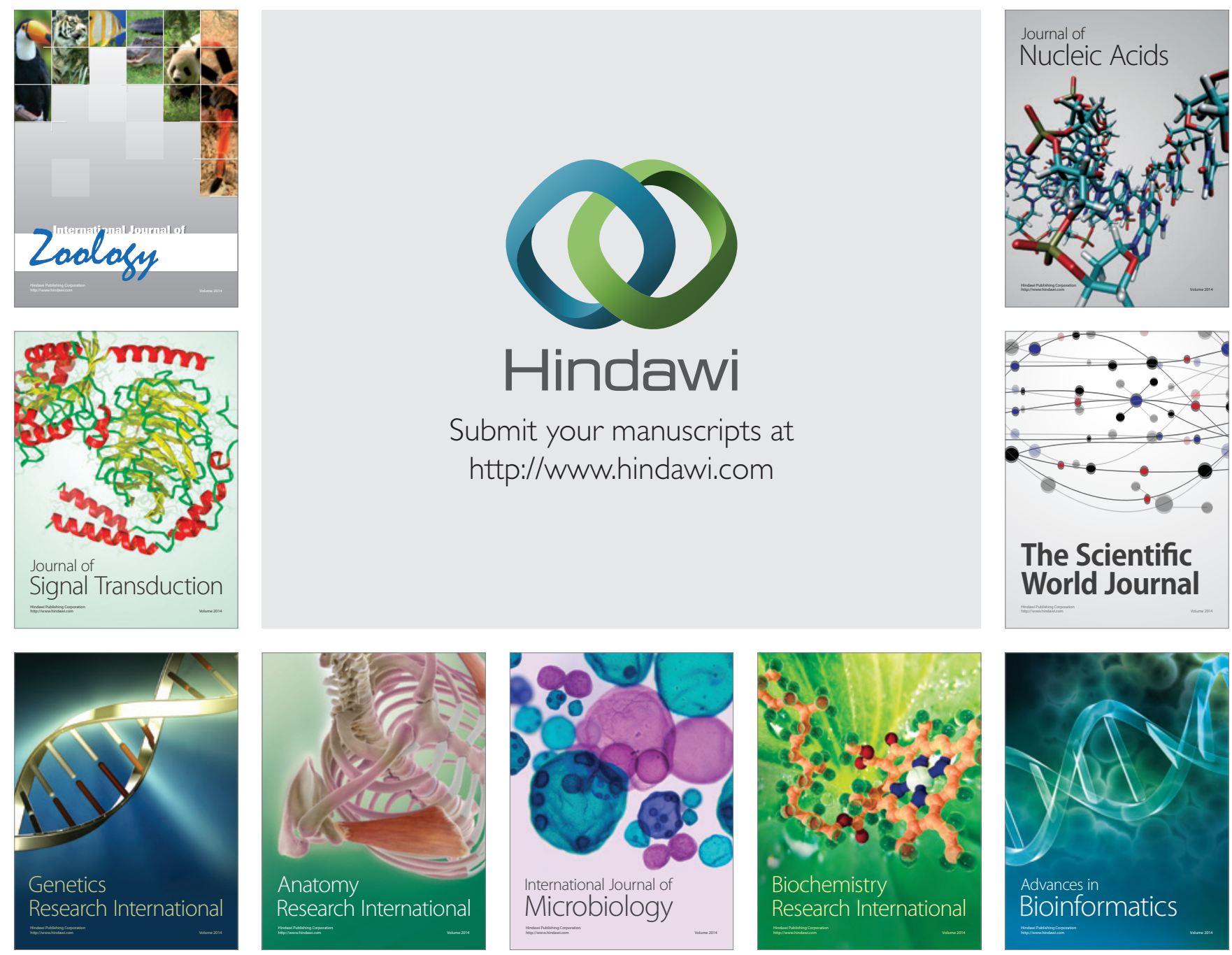

The Scientific World Journal
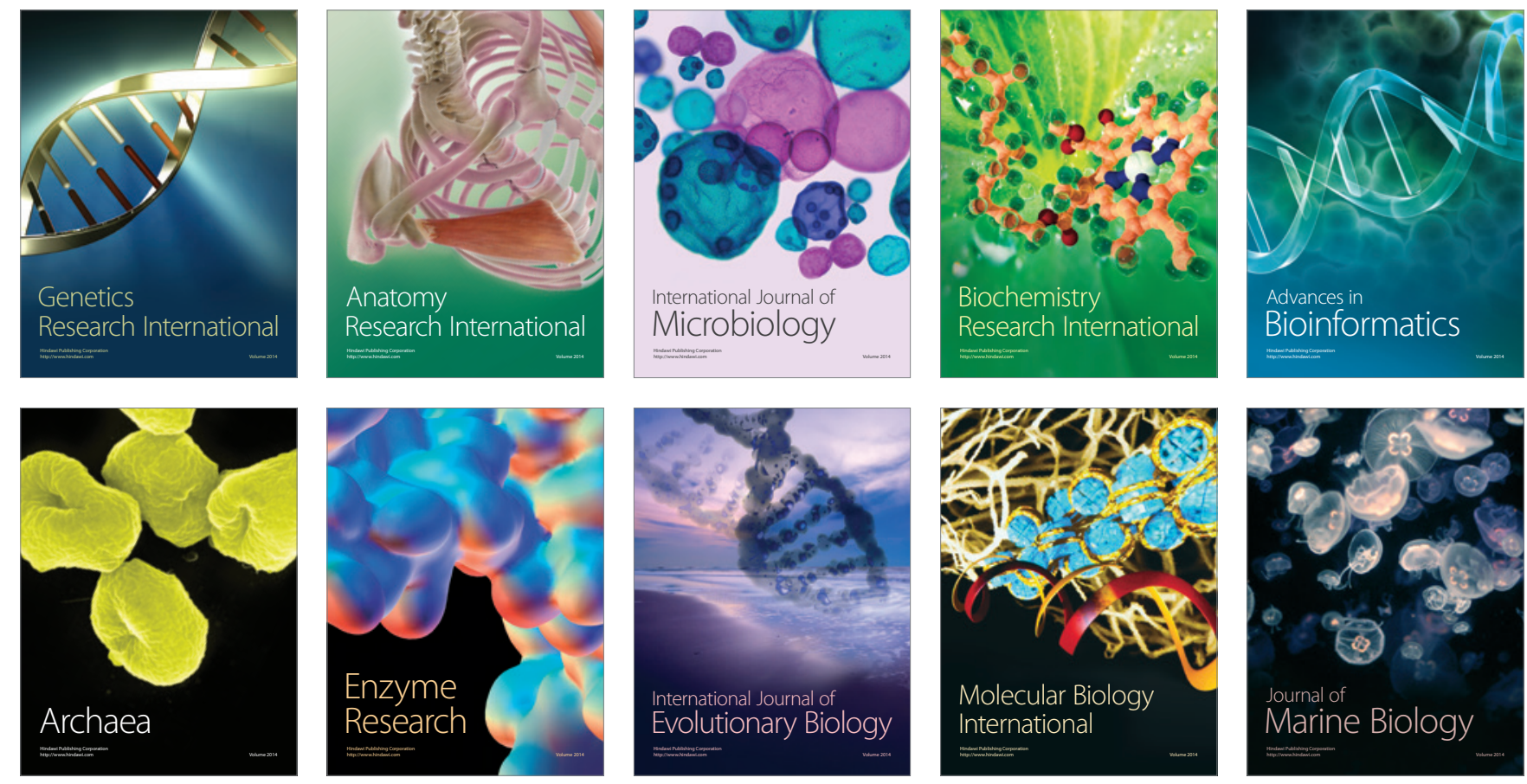